Original Research Article

\title{
A prospective observational study on incidence of adverse drug reactions in a tertiary care teaching hospital: a pharmacovigilance study
}

\author{
S. Sre Akshaya Kalyani ${ }^{1}$, Pendota Srihitha ${ }^{1}$, Katnapally Abhinay Sharma ${ }^{1}$, \\ Porandla Dharanija ${ }^{1}$, Sandeep Kumar Bheemreddy ${ }^{2}$
}

\begin{abstract}
${ }^{1}$ Department of Clinical Pharmacy, ${ }^{2}$ Department of Clinical Pharmaceutics, Vaageswari College of Pharmacy, Karimnagar, Telangana, India
\end{abstract}

Received: 02 August 2019

Revised: 23 September 2019

Accepted: 01 October 2019

\section{*Correspondence to:}

Dr. S. Sre Akshaya Kalyani, Email: akshaya273@gmail.com

Copyright: (C) the author(s), publisher and licensee Medip Academy. This is an openaccess article distributed under the terms of the Creative Commons Attribution NonCommercial License, which permits unrestricted noncommercial use, distribution, and reproduction in any medium, provided the original work is properly cited.

\begin{abstract}
Background: An adverse drug reaction (ADRs) is determined as response to a drug that is noxious unintended excludes therapeutic failures, overdose, drug abuse, noncompliance, and medication errors. The main aim of the study is to detect, understand and report ADR'S.

Methods: This study is prospective observational study conducted for 6 months in in-patient setting in a tertiary care hospital. Naranjo's, WHO causality scale, Siegel scale, Schumock and Thornton scale are used to assess ADR. Graph Pad Prism and SAS software's are used.

Results: Data was collected from a total of 1000 patients of which $121(12.1 \%)$ patients were effected with 150 ADRs. Among 121 patients AdrAd was 60.66\% and AdrIn was $39.33 \%$. Of 121 patients 97 patients with single ADR, 28 patients with 2 ADRs, 10 patients were with three ADRs. ADR onset divides acute $(10 \%)$, Latent $(39 \%)$ and sub-acute $(51 \%)$. ADR occurred are recovered $(54 \%)$, Recovering (13\%). Naranjos scale interprets definite $(0.9 \%)$, probable $(50.9 \%)$, possible $(42.97 \%)$. According to WHO scale certain $(2.7 \%)$, unlikely $(2.7 \%)$, possible (38.84\%). Hartwig and Siegel scale results are mild (12.4\%), moderate $(66.12 \%)$ and severe $(12.4 \%)$. Schumock and Thornton preventability results are definitely $(25.45 \%)$, probably $(68.18 \%)$ and not preventable $(6.36 \%)$. Conclusions: Every health care professional should be aware of the Pharmacovigilance principles and also should be aware of suspected ADR reporting form of PVPI. By applying the above scales it is easy for health care professionals to assess an ADR.
\end{abstract}

Keywords: Adverse drug reaction, Adverse drug reaction reporting form, Naranjo's and WHO causality assessment scales, Hartwig and Seigel scale, ADR incidence, CDSCO reporting form

\section{INTRODUCTION}

World Health Organisation (WHO) defines an adverse drug reaction (ADR) as "response to a drug that is noxious unintended and that occurs at doses used in humans for prophylaxis, diagnosis, or therapy of disease, or for the modification of physiologic function" excludes therapeutic failures, overdose, drug abuse, noncompliance, and medication errors. ${ }^{1}$
The epidemiology of ADRs in the Indian population is not known as very few studies have been reported. ADRs are 4th to 6th leading cause of death among hospitalized patients and has $6.7 \%$ incidence rate for serious ADRs. ${ }^{2}$ ADR is leading to $0.3 \%$ to $7 \%$ of all hospital admissions which is leading to annual dollar costs in the billions. $30 \%$ to $60 \%$ of them are preventable. ${ }^{3}$ There are different types of classifications of ADRs based on onset of event, severity of reaction and type of reaction. ${ }^{3-5}$ 


\section{ADR detection and management}

- Subjective report: Including patient complaints

- Objective report: Direct observation of event and abnormal findings including (physical exam, laboratory test, and diagnostic procedure).

- Medication order screening: abrupt medication discontinuation; abrupt dosage reduction; orders for tracer or trigger substances; orders for special tests or serum drug concentrations

- $\quad$ Spontaneous reporting

- Medication utilization review: Computerized screening; Chart review and concurrent audits. ${ }^{6-9}$

\section{Management options}

- Discontinue the offending agent

- Continue the medication if: it is medically necessary

- Discontinue non-essential medications

- Administer appropriate symptomatic treatment

- Provide supportive or palliative care (e.g, hydration, glucocorticoids, warm / cold compresses, analgesics or antipruritics)

- Consider re-challenge or desensitization. ${ }^{10-13}$

\section{Objectives}

The main objective of this study is to identify ADRs and their incidence in hospitalized patients and to report the ADRs to CDSCO and UMC. This study reveals the incidence and prevalence rates of $\mathrm{ADR}$ and to find out new ADR's.

\section{METHODS}

This study is a prospective, observational study conducted for 6 months among patients admitted into inpatient setting in Chelmada Anand Rao Institute of Medical Sciences which is a 1000-bedded hospital located in Karimnagar, Telanagana. The study took place between august 2017 and January 2018.

\section{Study population}

The participants of the study were taken from patients who were admitted to the in-patient setting of Chelmada Anand Rao Institute of Medical Sciences (tertiary care hospital) with ADR. All the patients provided consent for the study.

\section{Selection criteria}

Inclusion criteria were patients above age of 14 years; only patients who are hospitalized during study period; patients of both genders.

Exclusion criteria were patients of less than 14 years of age; patients who are admitted in oncology department; women who are admitted in gynaecology department; immunocompromised patients; patients with multiple comorbidities and those taking multiple medications $(>5$ medicines) for longer period of time; patients developing ADRs due to fresh blood or blood products infusion or accidental poisoning.

\section{Data collection}

Case records of all the patients were observed and the following data was obtained from each patient:

- Demographic details- age, sex

- Complaints of admission and present illness

- Medical and medication history

- Any allergies previously present

- Habits- type of diet, smoking, alcohol consumption

- Laboratory findings-hemogram, serum biochemistry, blood pressure.

ADR is suspected using ADR scales i.e, Naranjo's and WHO scale. The obtained ADRs are categorized using severity and preventability scale. It is also differentiated as occurrence of ADR due to hospital admission and ADR as a cause of hospital admission.

In case of absence of history of ADR or no data regarding ADR in case report then the patient is interviewed and the patient's medication consumption behaviour is evaluated.

\section{Reporting}

The ADRs obtained were filled in CDSCO (central drug standards for control of organisation) form which is according to WHO-UMC and are reported time to time to Pharmacovigilance programme of India (PVPI). ${ }^{8,28}$

\section{Statistical analysis}

The data was analysed using Microsoft-Excel and Graph Pad Prism 7.0. The ADRs were assessed significantly using chi-square and T-Test. SAS 9.2 is also used.

\section{RESULTS}

\section{Incidence of $A D R s$}

Data of $1000(n=1000)$ hospitalized patient population have been collected and results have been interpreted. Out of 1000 patients $150(15 \%)$ ADRs were detected in 121 $(12.10 \%)$ patients. Incidence rate of ADRs was found to be $12.1 \%$.

\section{Differentiation of ADR in occurrence}

ADR occurrence is differentiated into two types i.e, ADR occurred may lead to hospitalization (AdrAd), ADR 
occurred after hospitalization (AdrIn). Out of 121 patients $60.66 \%$ (91) of population had been presented with ADR after hospitalization while ADR lead to hospitalization in $39.33 \%$ (59) of population.

\section{Gender distribution of ADRs}

ADRs are differentiated gender wise. Of 1000 patients female patients were $42.3 \%$ (423 with S.D of $47.01 \pm 17$ ) and male patients were $57.7 \%$ (577 with S.D of $48.183 \pm 18)$, in which $66(54.54 \%$ with S.D of $42.8 \pm 15.3)$ females and $55(45.45 \%$ with S.D of $45.3 \pm 15.9)$ males were affected with ADRs respectively. Gender distribution in our study shows that females are more affected with ADRs than males.

\section{Age group wise distribution of ADRs.}

As per the inclusion criteria, only age groups of 15 and above patients were studied. Of which 31-45 (33.8\%) age group individuals are mostly affected with ADRs followed by $46-60(26.45 \%)$. As patients of 75-90 age group admitted in hospital were less in number compared to other age groups. So, the prevalence of ADR in those groups is also less. Chi-square test was performed and the $\mathrm{P}$ value was found to be $<0.0001$ at 4 degrees of freedom. Chi-square test it proves that there is a significant difference in the ADR distribution in age group.

\section{Distribution of ADRs in patients}

Among 121 patients with ADRs there are 150 ADRs as mentioned in Figure 3.5.2. Patient with single ADR are 9.2\% (92), patients with two ADRs include 1.4\% (14), and three ADRs include 1\% (10).

\section{Classification of ADRs based on onset}

Based on onset ADRs were classified into acute, subacute and latent. Of which $51 \%$ (74) of the patients were shown with sub-acute type of ADR's i.e, most of the ADR's were seen within 1-24hrs. 39\% (56) of the patients were with latent type of ADR's and 10\% (15) of the patients were with acute type of ADR's.

\section{Assessment of ADR outcome}

ADR outcome is classified into different types. 54\% (80) of the population were recovered from the ADR, $30 \%$ (45) of the population are not recovered while $13 \%$ (20) of the population are recovering and 3\% (5) of them are unknown which is due to unavailability of follow up.

\section{Assessment of Naranjo's causality scale}

According to Naranjo's causality assessment scale, probable and possible ADRs were with $50.90 \%$ (56) and $42.97 \%$ (52), whereas definite and doubtful were observed less because for most of the ADRs serum levels estimation, Re-challenge were not performed.

\section{Assessment of WHO-UMC causality scale}

For assessing causality WHO-UMC scale is also used. According to WHO scale the results were certain $2.7 \%$ (03), possible $38.84 \%$ (47), probable $47.11 \%$ (57), unlikely $2.70 \%$ (3) unclassified (0) and unassessable (0). Most of the ADRs were probable followed by possible as mentioned in Table 1.

Table 1: WHO-UMC scale interpretation.

\begin{tabular}{|lll|}
\hline WHO scale & Score & Interpretation $(\%)$ \\
\hline Certain & 3 & 2.70 \\
\hline unlikely & 3 & 2.70 \\
\hline possible & 47 & 38.84 \\
\hline probable & 57 & 47.11 \\
\hline Unclassified & 0 & 0 \\
\hline Unassessable & 0 & 0 \\
\hline Total & 110 & \\
\hline
\end{tabular}

\section{Assessment of Hartwig and Siegel severity scale}

For assessing severity of ADR, Hartwig \& Siegel severity scale was used. Individual levels of the scale are assessed and interpreted. According to Hartwig \& Siegel severity assessment scale moderate ADRs were high i.e, 66.12\% (80), mild and severe were found to be $12.4 \%$ (15) in Figure 1.

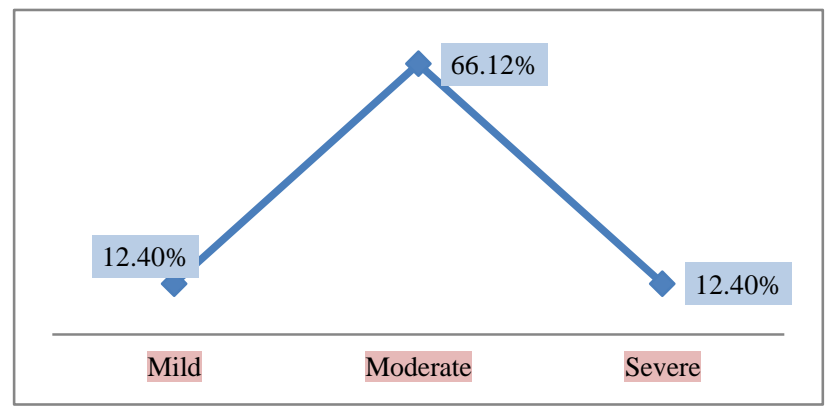

Figure 1: Hartwig and Siegel severity assessment scale interpretation.

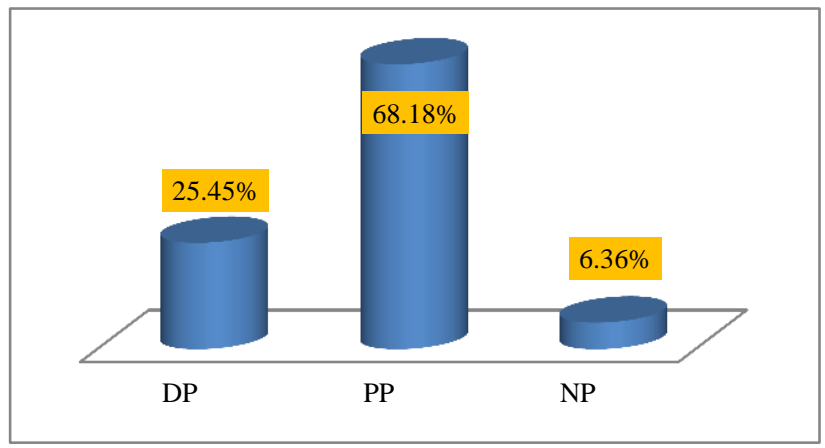

Figure 2: Graphical representation of Schumock and Thornton preventability scale. 


\section{Assessment of Schumock and Thornton preventability} scale

According to Schumock and Thornton preventability scale probably preventable (PP) ADRs were $68.18 \%$, definitely preventable (DP) ADRs were $25.45 \%$ and Not Preventable (NP) ADRs were $6.36 \%$ as mentioned in Figure 2.

\section{Incidence of drug classes causing ADRs}

Among different classes of drugs, anti-leprotic agents were found to have more risk of causing ADR i.e, of $1.1 \%$ patients used anti-leprotics $18.18 \%$ of them had a risk of causing ADR. Other drugs include antiepileptics+ anxiolytics (6.20\%), opiod analgesics (5.46\%), corticosteroids $(3.89 \%)$, antibiotics $(3.54 \%)$ supplements (2.61\%), anti-hypertensives (2.27\%), NSAIDS $(2.17 \%)$, anti-diabetic $(1.69 \%)$, antimalarial $(1.19 \%)$, respiratory drugs $(1.02 \%)$, gastric acid suppressants $(0.85 \%)$, antiemetics $(0.19 \%)$ as mentioned in Figure 3. According to Chi-Square test $(p<0.005)$ there is a significant different between the total population effected and ADR population.

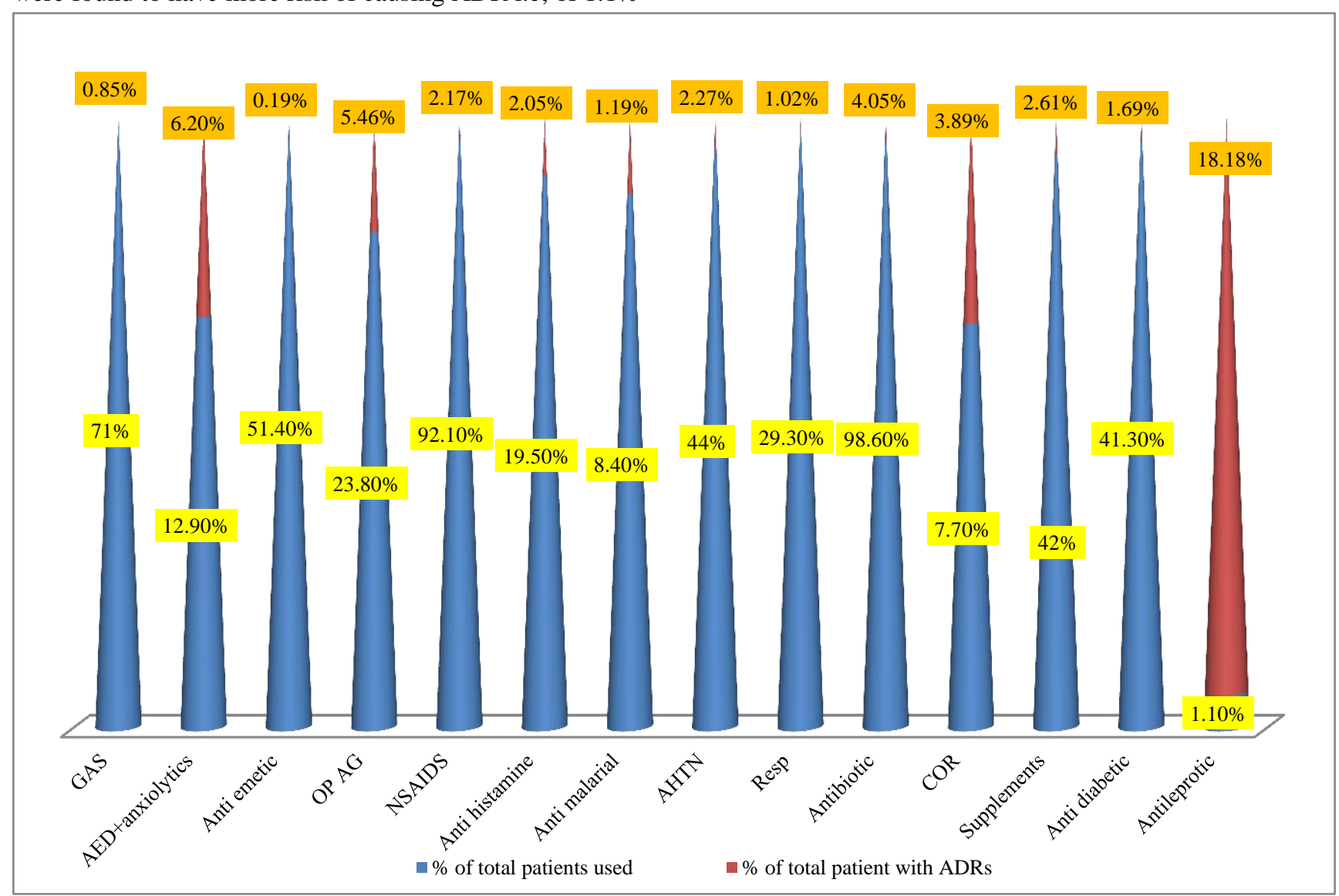

Figure 3: Prevalence of drug classes causing ADRs.

\section{Organ based classification of ADRs}

By categorizing the ADRs into different organ systems, most of the ADRs were gastrointestinal (38\%) followed by dermatological $(23.30 \%)$ then metabolic $(9.33 \%)$, CNS (8\%), CVS $(7.30 \%)$, hepatic $(5.30 \%)$, renal $(4 \%)$ and musculoskeletal $(1.33 \%)$.

\section{Classification of ADR type based on Rawlins and Thompson classification}

According to Rawlins and Thompson classification ADRs were classified in to type A, B, C, D, E. Type A ADRs were mostly seen with $65.54 \%$ in this study followed by type C (20.27\%), type B (14.18\%) as mentioned in Figure 4.

\section{Incidence of antibiotic classes}

Antibiotics causing ADRs were categorized in to different classes of antibiotics among them cephalosporins were mostly used with ADR incidence of $4.72 \%$ and rifamycins are with highest incidence $13.30 \%$ followed by tetracyclines $6.77 \%$, nitroimidazole $3.33 \%$, macrolide and floroquinolone are with $2.12 \%$ of incidence rate as mentioned in figure 3. Kolmogorov-Smirnov test (0.667) determines that there is a significant difference $(\mathrm{p}=0.0336)$ between population used antibiotics and population effected with ADRs. 


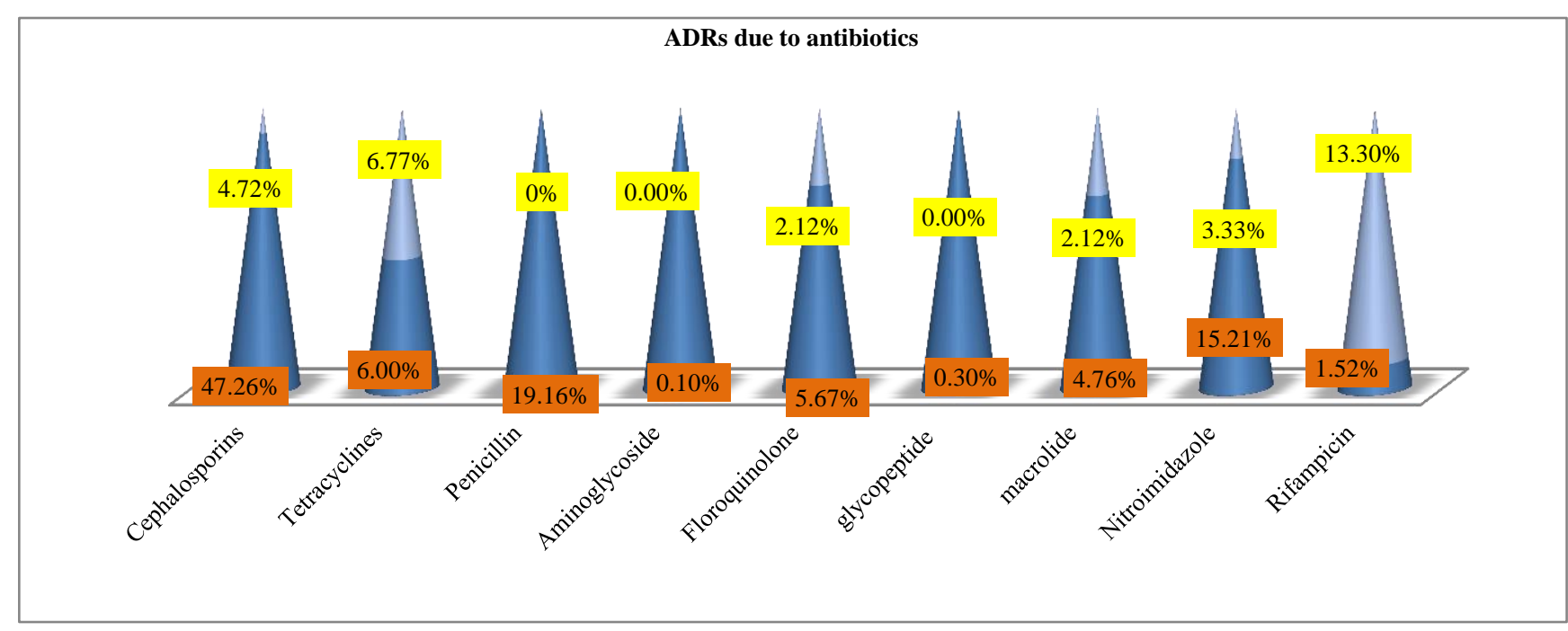

Figure 3: Antibiotics incidence of individual ADR.

Table 2: Representing some drug classes with individual drug ADRs observed in this study.

\begin{tabular}{|c|c|c|c|}
\hline Drug classes & $\begin{array}{l}\text { No. of } \\
\text { ADR's }\end{array}$ & $\begin{array}{l}\text { Drugs (no. of ADR's for each } \\
\text { drug) }\end{array}$ & ADR observed \\
\hline $\begin{array}{l}\text { Antiepileptic+ } \\
\text { anxiolytics }\end{array}$ & 10 & $\begin{array}{l}\text { a) Midazolam (1) } \\
\text { b) Phenytoin ( } 7 \text { ) } \\
\text { c) Carbamazepine (2) }\end{array}$ & $\begin{array}{l}\text { a) Reduced blood pressure } \\
\text { b) Cerebellar ataxia, dress syndrome, nystagmus, } \\
\text { multiple tissue connective disorder, dysarthria, } \\
\text { thrombocytopenia, headache } \\
\text { c) SJS, maculo popular rash }\end{array}$ \\
\hline GAS & 5 & $\begin{array}{l}\text { a) Pantoprazole (3) } \\
\text { b) Ranitidine (1) } \\
\text { c) Racecodotril (1) }\end{array}$ & $\begin{array}{l}\text { a) Constipation, loose stools, rashes over abdomen } \\
\text { and over both hands } \\
\text { b) QT interval prolongation with Flutter fibrillation } \\
\text { pattern } \\
\text { c) Increased blood pressure }\end{array}$ \\
\hline Antiemetic & 1 & a) Metaclopromide (1) & a) Headache \\
\hline Antibiotics & 40 & $\begin{array}{l}\text { a) Cefuroxime (1) } \\
\text { b) Doxycycline (4) } \\
\text { c) Cefixime (2) } \\
\text { d) Cefpodoxime (1) } \\
\text { e) Metronidazole (4) } \\
\text { f) Ceftriaxone (16) } \\
\text { g) Amoxicillin+Clavulanic acid (3) } \\
\text { h) Ofloxacin (1) } \\
\text { i) Ceftizoxime (1) } \\
\text { j) Ampicillin (2) } \\
\text { k) Ciprofloxacin (2) } \\
\text { l) Rifampicin (2) } \\
\text { m) Azithromycin }\end{array}$ & $\begin{array}{l}\text { a) Nausea, vomiting } \\
\text { b) Nausea, vomiting, papular lesions over neck, } \\
\text { papular lesions on body, loose motions } \\
\text { c) Erythematous vesicular crusts over face, oral } \\
\text { erosions, jaundice } \\
\text { d) SJS } \\
\text { e) Vomiting, nausea, diarrhea } \\
\text { f) Vomiting, loose motions, rashes, itching, malena } \\
\text { acute generalised exanthamaotous pustulosis, } \\
\text { eosinophilia } \\
\text { g) Nausea,vomiting, ithcing over the body, rashes } \\
\text { pyridoxine deficiency, } \\
\text { h) Acute utricaria } \\
\text { i) Tingling of both limbs } \\
\text { j) Diarrhea } \\
\text { k) Diarrhea, FDE } \\
\text { l) Increased Liver function tests, Hepatitis } \\
\text { m) Hyperpigmentation macules, papules over limbs }\end{array}$ \\
\hline $\begin{array}{l}\text { Anti } \\
\text { inflammatory }\end{array}$ & 22 & $\begin{array}{l}\text { a) Paracetamol (14) } \\
\text { b) Diclofenac (4) } \\
\text { c) Aceclofenac (1) } \\
\text { d) Prednisolone (2) } \\
\text { e) Others (1) }\end{array}$ & $\begin{array}{l}\text { a) Itching, rashes, diarrhea } \\
\text { b) Loose motions, hyperpigmented patches over } \\
\text { abdomen and groin, urosepsis with chronic kidney } \\
\text { disease, fixed drug eruption } \\
\text { c) Nephropathy } \\
\text { d) Nausea } \\
\text { e) AKI }\end{array}$ \\
\hline
\end{tabular}




\begin{tabular}{|c|c|c|c|}
\hline Drug classes & $\begin{array}{l}\text { No. of } \\
\text { ADR's }\end{array}$ & $\begin{array}{l}\text { Drugs (no. of ADR's for each } \\
\text { drug) }\end{array}$ & ADR observed \\
\hline $\begin{array}{l}\text { Anti- } \\
\text { hypertensive }\end{array}$ & 22 & $\begin{array}{l}\text { a) Enalapril (1) } \\
\text { b) Hydrochlorthiazide (2) } \\
\text { c) Telmisartan+ Hydrochlorthiazide } \\
\text { (1) } \\
\text { d) Metoprolol (1) } \\
\text { e) Telmisartan (4) } \\
\text { f) Atenolol (1) } \\
\text { g) Amlodipine (3) } \\
\text { h) Chlorthalidone+Telmisartan (2) } \\
\text { i) Cilnidipine (1) }\end{array}$ & $\begin{array}{l}\text { a) AKI } \\
\text { b) Vertigo } \\
\text { c) Vomting, diarrhea } \\
\text { d) Bradycardia } \\
\text { e) Diarhhea } \\
\text { f) AKI } \\
\text { g) Loose motions, parkinsonism, edema } \\
\text { h) Hypochloremia, hyponatremia } \\
\text { i) Hypotension }\end{array}$ \\
\hline Anti-diabetic & 8 & $\begin{array}{l}\text { a) Metformin (5) } \\
\text { b) Glimepride (1) } \\
\text { c) Insulin (2) }\end{array}$ & $\begin{array}{l}\text { a) Hypoglycemia induced seizures } \\
\text { b) Hypoglycemic seizures } \\
\text { c) Hyperkalemia }\end{array}$ \\
\hline Analgesic & 23 & $\begin{array}{l}\text { a) Tramadol (22) } \\
\text { b) Others (1) }\end{array}$ & $\begin{array}{l}\text { a) Vomiting, nausea, headache, dryness of mouth } \\
\text { b) Hyperpigmentation over back and front of chest } \\
\text { wall }\end{array}$ \\
\hline
\end{tabular}

Table 3: WHO-UMC numbers for ADR.

\begin{tabular}{|c|c|c|c|}
\hline S.No. & Patient details & Adverse reaction & WHO-UMC number \\
\hline 1. & SR-70/M & Diclofenac induced urosepsis & $2018-00865$ \\
\hline 2. & CK-28/F & Paracetamol induced rash & 2018-00879 \\
\hline 3. & MC-64/M & Ranitidine induced syncopeal attack & $2018-00896$ \\
\hline 4. & MUK-46/M & Paracetamol induced rashes & 2018-00903 \\
\hline 5. & RB-29/M & Prednisolone induced erythematous rash & 2018-01169 \\
\hline 6. & RK-35/F & Cefixime and paracetamol induced loose motions & 2018-01184 \\
\hline 7. & SS-43/M & Tramadol induced vomiting's & 2018-01190 \\
\hline 8. & PR-58/F & pantoprazole induced constipation & 2018-01193 \\
\hline 9. & PR-57/M & Midazolam induced hypotension & 2018-01204 \\
\hline 10. & PR-57/M & meropenem induced hypokalemia & 2018-01207 \\
\hline 11. & $\mathrm{SS}-43 / \mathrm{M}$ & Baclofen induced constipation & 2018-01215 \\
\hline 12. & SURI-29/F & Lumerax induced vomiting's & 2018-01807 \\
\hline 13. & SUR-29/F & Ceftriaxone and pantoprazole induced loose motions & 2018-01810 \\
\hline 14. & $\mathrm{SH}-40 / \mathrm{M}$ & Prednisolone induced nausea & 2018-02132 \\
\hline 15. & $\mathrm{EK}-47 / \mathrm{F}$ & Tramadol induced vomiting's & $2018-02141$ \\
\hline 16. & OS-43/M & Metformin induced hypoglycemic seizures & 2018-02171 \\
\hline 17. & $\mathrm{AS}-74 / \mathrm{F}$ & Ampicillin+sulbactam, pantoprazole induced loose motions & $2018-02172$ \\
\hline 18. & $\mathrm{BS}-45 / \mathrm{F}$ & Metformin; Glibenclamide induced seizures & $2018-02185$ \\
\hline 19. & MM-60/M & Pantoprazole induced loose motions & 2018-02186 \\
\hline 20. & GV-27/F & $\begin{array}{l}\text { Ondansetron, ceftriaxone and doxycycline induced loose } \\
\text { motions }\end{array}$ & 2018-03035 \\
\hline 21. & DR-49/M & Carbamazepine induced SJS & 2018-03141 \\
\hline 22. & $\mathrm{RM}-64 / \mathrm{M}$ & $\begin{array}{l}\text { Ciprofloxacin, dexamethasone and diclofenac induced } \\
\text { maculopapular rash }\end{array}$ & $2018-03150$ \\
\hline 23. & BR-28/F & Phenytoin induced DRESS & $2018-03360$ \\
\hline 24. & SY-60/F & Amlodipine induced Parkinsonism & 2018-03374 \\
\hline 25. & PNE-58/M & Dapsone, Hansepran induced Hemolytic Anemia & 2018-03929 \\
\hline 26. & $54 / \mathrm{F}$ & $\begin{array}{l}\text { Ceftriaxone, } \\
\text { metronidazole }\end{array} \quad$ Nausea vomiting & 2018-07125 \\
\hline 27. & 38/M & Loose stools & 2018-07164 \\
\hline 28. & $22 / \mathrm{M}$ & Nausea vomiting & $2018-07170$ \\
\hline 29. & $22 / \mathrm{M}$ & Tranexamic acid & $2018-07467$ \\
\hline 30. & $60 / \mathrm{F}$ & Cerebellar ataxia & 2018-10108 \\
\hline 31. & $52 / \mathrm{F}$ & Itching hyper pigmented macules & $2018-07489$ \\
\hline
\end{tabular}




\begin{tabular}{|c|c|c|c|c|}
\hline S.No. & Patient details & Adverse reaction & & WHO-UMC number \\
\hline 32. & $31 / \mathrm{M}$ & $\begin{array}{l}\text { Amoxicillin, } \\
\text { Tramadol }\end{array}$ & Nausea vomiting & 2018-07513 \\
\hline 33. & $5 / \mathrm{F}$ & Ofloxacin & Acute urticarial & 2018-07564 \\
\hline 34. & $54 / \mathrm{M}$ & $\begin{array}{l}\text { Docycycline, } \\
\text { Ceftriaxone }\end{array}$ & Papular lesions & 2018-07748 \\
\hline 35. & $48 / \mathrm{F}$ & $\begin{array}{l}\text { Tramadol+Paraceta } \\
\text { mol }\end{array}$ & Vomiting's & 2018-07712 \\
\hline 36. & $19 / \mathrm{F}$ & Methotrexate & Renal failure & 2018-07808 \\
\hline 37. & $51 / \mathrm{F}$ & Aceclofenac & Nephropathy & 2018-07823 \\
\hline 38. & $51 / \mathrm{F}$ & Thyroxine & Muscle weakness & 2018-07839 \\
\hline 39. & $23 / \mathrm{F}$ & Paracetamol, Caripil & Itching, rash & 2018-07849 \\
\hline 40. & $23 / \mathrm{F}$ & $\begin{array}{l}\text { Paracetamol, } \\
\text { amoxicillin }\end{array}$ & Itching & 2018-08106 \\
\hline 41. & $55 / \mathrm{M}$ & Doxycycline, Caripil & Nausea vomiting & $2018-08126$ \\
\hline 42. & $44 / \mathrm{M}$ & Diclofenac & Itching, lesions & $2018-08665$ \\
\hline 43. & $43 / \mathrm{F}$ & $\begin{array}{l}\text { Ceftriaxone, } \\
\text { pantoprazole }\end{array}$ & Loose stools & 2018-08687 \\
\hline 44. & $44 / \mathrm{F}$ & Enalapril & Acute kidney injury & 2018-08751 \\
\hline 45. & $42 / \mathrm{F}$ & $\begin{array}{l}\text { Ciprofloxacin, } \\
\text { metronidazole }\end{array}$ & Loose stools & 2018-08818 \\
\hline 46. & $50 / \mathrm{F}$ & Paracetamol & Itching & 2018-08830 \\
\hline 47. & $30 / \mathrm{F}$ & Cefpodoxime & Hyper pigmented macules & 2018-08839 \\
\hline 48. & $60 / \mathrm{F}$ & Dextrose/ insulin & Hyperkalemia & $2018-11230$ \\
\hline 49. & $31 / \mathrm{M}$ & Tramadol & Vomiting & 2018-11272 \\
\hline 50. & $44 / \mathrm{M}$ & $\begin{array}{l}\text { Tramadol/metronida } \\
\text { zole }\end{array}$ & Nausea and vomiting & 2018-11274 \\
\hline 51. & $3 / \mathrm{F}$ & Iron & Black colored stools & 2018-11406 \\
\hline 52. & $58 / \mathrm{F}$ & Tramadol & Dryness of mouth & 2018-11437 \\
\hline 53. & $64 / \mathrm{F}$ & Sodium bicarbonate & Hypernatremia & 2018-11444 \\
\hline 54. & $66 / \mathrm{F}$ & $5 \mathrm{fu}$ & Oral ulcers, neutropenia anemia & 2018-11525 \\
\hline 56. & $2 / \mathrm{F}$ & Ceftriaxone & Rashes & 2018-11446 \\
\hline 57. & $66 / \mathrm{F}$ & Oxaliplatin & Swelling over tongue, pain & 2018-11449 \\
\hline 58. & $11 / \mathrm{F}$ & $\begin{array}{l}\text { Ferrous } \\
\text { ascorbate/folic acid }\end{array}$ & Black colored stools & 2018-11531 \\
\hline 59. & $64 / \mathrm{M}$ & Ranitidine & QT Prolongation & 2018-11549 \\
\hline 60. & $43 / \mathrm{M}$ & $\begin{array}{l}\text { Benzoate, } \\
\text { Doxofyllin }\end{array}$ & Vomiting's & 2018-11554 \\
\hline 61. & $28 / \mathrm{F}$ & $\begin{array}{l}\text { Tramadol, } \\
\text { amoxicillin }\end{array}$ & Nausea and Vomiting's & $2018-11651$ \\
\hline 62. & $10 \mathrm{~m} / \mathrm{M}$ & Paracetamol & Vomiting's & $2018-11572$ \\
\hline 63. & $41 / \mathrm{F}$ & Fexofenadine & Vomiting's & $2018-11655$ \\
\hline 64. & $45 / \mathrm{M}$ & $\begin{array}{l}\text { Ceftriaxone, } \\
\text { doxycycline }\end{array}$ & Papular lesions & 2018-11664 \\
\hline 65. & $33 / \mathrm{F}$ & Tramadol & Vomiting's & 2018-11672 \\
\hline 66. & $55 / \mathrm{M}$ & Metformin & Hypoglycemia & 2018-11793 \\
\hline 67. & $69 / \mathrm{F}$ & $\begin{array}{l}\text { Amlodipine, } \\
\text { Doxofylline }\end{array}$ & Loose stools & 2018-11804 \\
\hline 68. & $36 / \mathrm{F}$ & Neomercazole & Leucopenia & 2018-11809 \\
\hline 69. & $7 / \mathrm{M}$ & $\begin{array}{l}\text { Sulfadoxine, } \\
\text { Primarquine }\end{array}$ & Vomiting's & 2018-11814 \\
\hline 70. & $44 / \mathrm{F}$ & $\begin{array}{l}\text { Tramadol, } \\
\text { ceftriaxone }\end{array}$ & Vomiting's & 2018-11818 \\
\hline 71. & 29/M & Prednisolone & Pedal edema & 2018-11859 \\
\hline 72. & 45/M & Ceftriaxone & AGEP & 2018-11875 \\
\hline 74. & 20/M & Paracetamol & Rahes & 2018-11888 \\
\hline
\end{tabular}




\begin{tabular}{|lllll|}
\hline S.No. & Patient details & Adverse reaction & & WHO-UMC number \\
\hline 75. & $56 / \mathrm{M}$ & Insulin & Hypoglycemia & $2018-17142$ \\
\hline 76. & $45 / \mathrm{F}$ & Telma H & Vertigo & $2018-17150$ \\
\hline $\mathbf{7 7 .}$ & $45 / \mathrm{F}$ & Glimepiride & Hypoglycemic Seizures & $2018-17171$ \\
\hline $\mathbf{7 8 .}$ & $45 / \mathrm{F}$ & Telma H & Vomiting \& Diarrhea & $2018-17181$ \\
\hline $\mathbf{7 9}$. & $48 / \mathrm{F}$ & Paracetamol & Vomiting & $2018-17185$ \\
\hline $\mathbf{8 0 .}$ & $64 / \mathrm{M}$ & Diclofenac & Acute Kidney Injury & $2018-17196$ \\
\hline $\mathbf{8 1 .}$ & $60 / \mathrm{M}$ & Pantoprazole & Loose Motions & $2018-17203$ \\
\hline $\mathbf{8 2 .}$ & $72 / \mathrm{M}$ & Perinorm & Headache & $2018-17354$ \\
\hline $\mathbf{8 3 .}$ & $75 / \mathrm{M}$ & Tramadol & Vomiting & $2018-17398$ \\
\hline $\mathbf{8 4 .}$ & $70 / \mathrm{F}$ & Atenolol & Acute Kidney Injury & $2018-17600$ \\
\hline $\mathbf{8 5 .}$ & $70 / \mathrm{M}$ & Metoprolol & Bradycardia & $2018-17606$ \\
\hline $\mathbf{8 6 .}$ & $70 / \mathrm{M}$ & Diclofenac & Diarrhea & $2018-17615$ \\
\hline $\mathbf{8 7 .}$ & $69 / \mathrm{M}$ & Amlodipine & edema & $2018-17620$ \\
\hline $\mathbf{8 8 .}$ & $67 / \mathrm{M}$ & Metformin & Hypoglycemic Seizures & $2018-17765$ \\
\hline $\mathbf{8 9}$. & $66 / \mathrm{F}$ & fluorouracil & Mouth ulcers & $2018-17779$ \\
\hline $\mathbf{9 0}$ & $28 / \mathrm{F}$ & Tramadol & Vomiting & $2018-17795$ \\
\hline $\mathbf{9 1}$ & $29 / \mathrm{F}$ & Tramadol & Headache & $2018-17800$ \\
\hline
\end{tabular}

Sample patient pictures of ADRs collected.

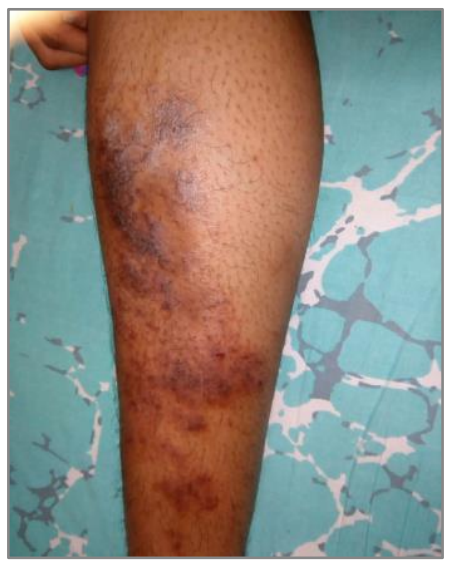

Figure 4: Carbamazepine induced Steven Johnsons syndrome.

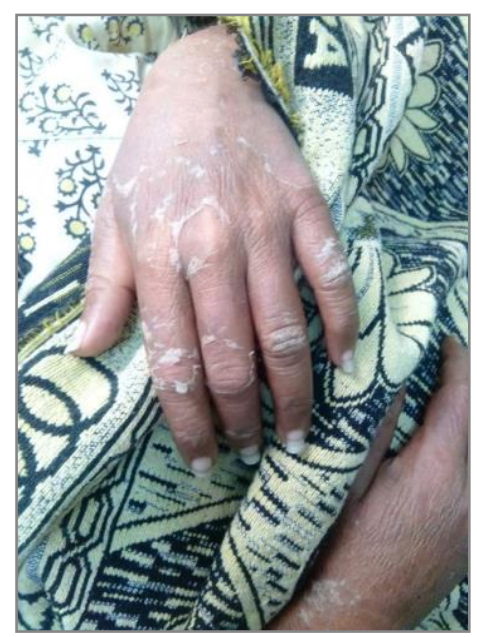

Figure 5: Carbamazepine induced maculopapular rash healing stage.

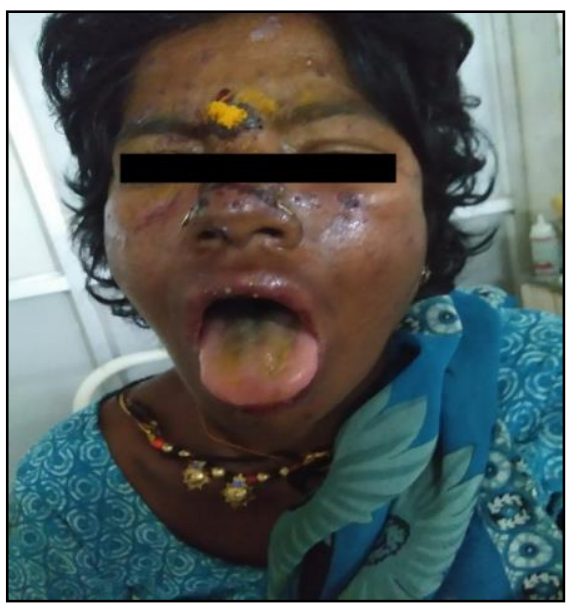

Figure 6: Cefixime induced erythematosus vesicular crusts over face, oral erosions.

\section{WHO-UMC numbers for ADR}

The following are list of individual case safety report (ICSRs) received from National coordinating committee (NCC) on 29 December 2017, 29 January 2018, 06 March 2018 which are reviewed and entry into vigiflow as mentioned in Table 3.

\section{DISCUSSION}

Adverse drug reactions adversely effect the health care system and health related quality of life of patients. The present study dealt with identifying, assessing and reporting of ADRs in a tertiary care hospital. According to our study the overall incidence rate of ADR was found to be $12.1 \%$. But this may differ across states and countries as the methodology adopted may be different. ${ }^{15-17}$

According to Tiwari et al, the incidence rate was also found to be $12 \%$ and males (75\%) were more significant 
than females $(25 \%)$. But according to our study females $(55 \%)$ are more significant than males $(45 \%)$. The age group 19-59 years showed with highest number of ADRs (60\%).This may differ depending the age group admitted in hospital. Also in his study GI (73\%) side effects were recorded as high followed by metabolic $(17 \%)$ whereas in our study also GI side effects (38\%) were high but followed by dermatologic reactions $(23.3 \%)$ and metabolic $(9.33 \%){ }^{21,22}$

According to Kharb, et al, males (66.33\%) are more prone to ADRs than that of females which in our case is opposite. GI cases were recorded high (31.43\%). Antimicrobials (43.37\%) had shown more ADRs followed by anticancer and immunosuppressive agents $(29.02 \%)$ which in our case these both categories are under exclusion criteria. Antimicrobials were also recorded high in our study $(28.92 \%)$. $^{23}$

According to Lihite, et al, 219 patients were reported with ADRs in which 73 patients were with single ADRs, 19 patients were with 3 ADRs and 3 patients were with 4 ADRs. $^{24,25}$ According to Tiwari et al 116 ADRs were recorded of which 56 patients were with 1 ADR and 60 patients were with more than 1 ADR. Comparing with our study out of 121 patients, 91 patients were with single ADRs, 14 patients were with 2 ADRs and 10patients were with 3 ADRs. According to him most of the ADRs were from dermatology department $(63.01 \%)$ followed by hematology $(18.26 \%)$ and psychiatry $(11.87 \%){ }^{21}$ According to some studies most of the ADRs were due to antibiotics.

According to Laskar, et al the ADR incidence rate was found to be $0.41 \%$ of which $0.22 \%$ of ADRs are AdrAd and $0.19 \%$ are AdrIn, which in our case out of $12 \%$ incidence $5.9 \%$ AdrAd was $5.9 \%$ and AdrIn was $7.28 \%{ }^{26}$

In order to assess causality, Naranjo's causality scale was used. According to Shamna et al, Naranjo scale revealed that $71.42 \%$ were probable, $18.36 \%$ were possible, $10.20 \%$ were definite and $0 \%$ were doubtful. ${ }^{27-29}$ But the study has limitations with re-challenge and estimation of serum drug concentrations. Our causality score includes $0.9 \%$ as definite, $50.9 \%$ are probable, $42.97 \%$ are possible and $0.9 \%$ are doubtful.

For assessing causality WHO-UMC scale is used. According to WHO scale the results were certain $(2.7 \%)$, possible $(38.84 \%)$, probable $(2.48 \%)$, unlikely $(47.11 \%)$, unclassified and unassessable were not ruled out. According to Singh et al, WHO-UMC results include certain $(9.74 \%)$, probable $(36.36 \%)$, possible $(31.16 \%)$, unlikely $(5.19 \%)$, unclassified $(7.79 \%)$ and unassessable $(9.74 \%)$.

Severity is assessed using Hartwig and Seigel severity assessment scale. According to Tiwari et al, mild severity $53.3 \%$ and moderate were $46.6 \%$. No ADR was found to be severe. In our study $12.4 \%$ were mild, $66.12 \%$ were moderate and $12.4 \%$ are severe. ${ }^{21}$

Schumock and Thornton scale is used to assess preventability of ADR. According to Padmavathi et al, Preventability assessment was $12.2 \%$ were definitely preventable and $87.8 \%$ were not preventable. ${ }^{38}$ According to our study $25.45 \%$ were definitely preventable, $68.18 \%$ were possibly preventable and $6.36 \%$ were not preventable. ${ }^{33,34}$ As the scale is not defined properly how to give a proper score, the inference of this scale may not be prompt.

According to Singh et al, Distribution of ADRs across therapeutic classes were Antimicrobials (28.57\%), antihypertensive's $(24.02 \%)$, anti-diabetics (14.28\%), and NSAIDs $(9.74 \%)^{30-32}$

According to Tiwari et al, gastrointestinal system (73\%) was found to be the most commonly affected organ system, followed by the metabolic (17\%), cutaneous system (5\%), haematological system (3\%) and cardiovascular system $(2 \%){ }^{21}$ In our study also it was quite evident that GI (38\%), ADRs are the highest followed by dermatologic (23.3\%), metabolic (9.33\%), CNS (8\%), CVS (7.3\%), renal (4\%) and musculoskeletal (1.33\%).

The study has fewer limitations. The Naranjo's scale used to assess causality has some limitations. Re-challenge and de-challenge are not done in most of the patients. Same limitations are also seen with the WHO causality scale. Schumock and Thornton preventability scale does not give a proper outcome, as the scale is not differentiated and interpreted properly. ${ }^{35,36}$

As there is less time period to assess large population with ADRs, so assessing each and every single ADR was a tough task because most of the patients were not included in the study due to various reasons such as no follow up of the patient, patients left under medical advice without treatment, intensive care patients were tough to assess with ADR and surgical department cases were with many number of drugs which lead to confusion of suspected drug.

\section{CONCLUSION}

Monitoring and reporting of ADRs must be an ongoing ceaseless and continuing process as new drugs are released into market all the time and their long term effects are not seen in clinical trials. Individualized effects, age group effects, conditional effects etc, cannot be observed during clinical trials. These are observed only in post marketing surveillance studies. Clinical pharmacist intervention in identifying ADRs is necessary in minimizing ADRs.

Our study revealed about the importance of identifying, assessing and reporting of ADRs. Every health care 
professional should be aware of the Pharmacovigilance principles and also should be aware of suspected ADR reporting form of PVPI. By applying the above scales it is easy for health care professionals to assess an ADR.

\section{ACKNOWLEDGEMENTS}

I express my deepest sense of thanks to my Dr. Ch. Pradeep Kumar, for his moral support and valuable suggestions at every instant. I specially thank Mr. Srinivas Velupula, Patient Safety Pharmacovigilance Associate (PVPI), ADR Monitoring Center-Department of Pharmacology, Kakatiya Medical College/MGM Hospital for his constant support in reporting ADRs.

\section{Funding: No funding sources}

Conflict of interest: None declared

Ethical approval: The study was approved by the Institutional Ethics Committee

\section{REFERENCES}

1. Parthasarathi G, Olsson S. Adverse drug reactions. In: Parthasarathi G, Karin, Hansen N, Nahata MC, editors. A Textbook of Clinical Pharmacy Practice: Essential concepts and skills, Inc; 2004: 84-102.

2. American Society of Hospital Pharmacists. ASHP Assistance Bulletin on Hospital Drug Distribution and Control. Am J Hosp Pharm. 1980;37:1097-103.

3. Malhotra S, Karan RS, Pandhi P, Jain S. Role of adverse drug reactions and non-compliance Postgrad Med J. 2001;77:703-7.

4. Calis KC. National Institutes of Health Clinical Analysis of Adverse Drug Reactions. Hospital Pharmacy. 2004;39(7):697-712.

5. Kessler DA. Introducing MedWatch, using FDA for 3500. A New Approach to Reporting Medication and Device Adverse Effects and Product problems. JAMA. 1993;269:2765-68.

6. Bates DW, Boyle DL, Vander Vliet MB, et al. Relationship between medication errors and adverse drug events. J Gen Intern Med. 1995;10:199-205.

7. Katzung BG, Trevor AJ Development and regulation of drugs. In: Katzung BG. Ed. Basic and Clinical Pharmacology. 10th edn. Lange Medical Books/McGraw-Hill, New York. 2007.

8. Murphy BM, Frigo LC. Development, implementation, and results of a successful multidisciplinary adverse drug reaction reporting program in a university teaching hospital. Hosp Pharm. 1993;28:1199-204.

9. Lazarou J, Pomeranz BH, Corey PN. Incidence of adverse drug reactions in hospitalized patients-a meta-analysis of prospective studies. JAMA. 1998;279:1200-5.

10. El-Eraky H, Thomas SHL. Effects of sex on the pharmacokinetic and pharmacodynamic properties of quinidine. Br J Clin Pharmacol. 2003;56:198-204.

11. Hermida RC, Ayala, DE, Calvo, C, Lopez, JE. Aspirin administered at bedtime, but not on awakening has an effect on ambulatory blood pressure in hypertensive patients. J Am Coll Cardiol. 2005;46:975-83.

12. Ahmed B, Nanji K, Mujeeb R, Patel MJ. Effects of polypharmacy on adverse drug reactions among geriatric outpatients at a tertiary care hospital in Karachi: a prospective cohort study. PloS One. 2014;17;9:e112133.

13. Rawlins MD, Thompson JW. Pathogenesis of adverse drug reactions. In: Davies DM, ed. Textbook of adverse drug reactions. Oxford: Oxford University Press; 1977: 10.

14. Arulmani R, Rajendran SD, Suresh B. Adverse drug reaction monitoring in a secondary care hospital in South India. Br J Clin Pharmacol. 2007;65:210-16.

15. Lang DM, Alpern MB, Visintainer PF, Smith ST. Increased risk for anaphylactoid reaction from contrast media in patients on beta-adrenergic blockers or with asthma. Ann Intern Med. 1991;115:270-76.

16. Petri M, Allbritton J. Antibiotic allergy in systemic lupus erythematosus: a case-control study. J Rheumatol. 1992;19:265-9.

17. Gholami K, Shalviri G. Factors Associated with Preventability, Predictability, and Severity of Adverse Drug Reactions. Ann Pharmacother. 1999;33:236-40.

18. Naranjo CA, Busto U, Sellers EM, Sandor P, Ruiz I, Roberts EA, et al. A method for estimating the probability of adverse drug reactions. Clin Pharmacol Ther. 1981;30:239-45.

19. World Health Organization (WHO). The Use of the WHO-UMC System for Standardised Case Causality Assessment. Geneva: WHO, 2014.

20. Hartwig SC, Siegel J, Schneider PJ Preventability and severity assessment in reporting adverse drug reactions. Am J Hosp Pharm. 1992;49:2229-32.

21. Tiwari P, Anuradha, D’Cruz S, Sachdev A Adverse Drug Reaction Monitoring in a North Indian Public Teaching Hospital. J Pharma Care Health Sys. 2016;3:164.

22. Amrinder R, Kaur I, Singh J, Kaur T, Monitoring of Cutaneous Adverse Drug Reactions in a Tertiary Care Hospital. J Pharmacovigilance. 2016;4:207.

23. Kharb P, Mittal N, Gu MC. An evaluation of adverse drug reactions monitoring at a Pharmacovigilance unit under Pharmacovigilance program of india in a tertiary care hospital of Haryana. Int $\mathbf{J}$ Basic Clin Pharmacol. 2015;4(3):556-60.

24. Lihite RJ, Lahkar M, Das S, Hazarika D, Kotni M, Maqbool M, et al. A Study on adverse drug reactions in a tertiary care hospital of northeast india, Alexandria J Med. 2017;53(2):151-6.

25. Laskar JI, Chakravarty P, Dewan B. A study on incidence of adverse drug reactions with commonly prescribed drugs and causality assessment in Silchar Medical College and Hospital, Int J Basic Clin Pharmacol. 2017;6(5):1175-83.

26. Hardmeier B, Braunschweig S, Cavallaro M, Roos M, Pauli-Magnus C, Giger M, et al. Adverse drug 
events caused by medication errors in medical inpatients. Swiss Med Wkly. 2004;134(45-46):66470 .

27. Shamna M, Dilip C, Ajmal M, Mohan PL, Shinu C, Jafer CP. A prospective study on adverse drug reactions of antibiotics in a tertiary care hospital, Saudi Pharm J. 2014;22(4):303-8.

28. Goyal RK, Bhatt PA, Burande MD. Adverse drug reactions and Pharmacovigilance: Elements of Clinical Pharmacy: published by S. B Shah, 3rd edition: 2006: 126-7.

29. Gohel D, Bhatt SK, Malhotra S. Evaluation of Dermatological Adverse Drug Reaction in the Outpatient Department of Dermatology at a Tertiary Care Hospital. Indian J Pharm Pract. 2014;7(3):42-9.

30. Singh H, Dulhani N, Kumar BN, Singh P, Tewari P, Nayak K. A Pharmacovigilance Study in Medicine Department of Tertiary Care Hospital in Chhattisgarh (Jagdalpur), India. J Young Pharm. 2010;2(1):95100 .

31. Suman A, Devesh, Gosavi D. Study of Adverse Drug Effects of Antiepileptic Drugs used in Pediatric Patients in a Tertiary care rural Hospital-a Pharmacovigilance Study. J Young Pharm. 2017;9(1):60-4.

32. Bhabhor PH, Patel TK, Vahora R, Patel PB, Desai N. Adverse drug reactions in a tertiary care teaching hospital in India: analysis of spontaneously reported cases, Int J Basic Clin Pharmacol. 2014;3(6):107885 .
33. Padmavathi S, Manimekalai K, Ambujam S. Causality, Severity and Preventability Assessment of Adverse Cutaneous Drug Reaction: A Prospective Observational Study in a Tertiary Care Hospital. J Clin Diagn Res. 2013;7(12):2765-67.

34. Gaur S, Paramjeet S, Srivastava B, Bhardwaj R, Ahuja S, Gunjita B. Evaluation of Adverse Drug Reactions in teaching hospital in Kumoun Region. JMSCR. 2016;4(8):12139-45.

35. Pendota S, Kalyani SSA, Katnapally AS, Porandla D, Bheemreddy S. Classification and applying pharmacovigilance principles to study adverse drug reaction and its management. Int $\mathrm{J}$ Basic Clin Pharmacol. 2017;6(11):2537-44.

36. Kalyani. SSA and Srihitha.P, An Epidemiological Study on Adverse Drug Reactions in Indian Population: Meta-Analysis. Int J Pharm Clin Res. 2017;9(10):654-9.

37. Kalyani SSA, Pendota S, Katnapally AS, Porandla D, Bheemreddy S. Cross sectional study on prevalence and medication adherence of hypertension and diabetes in a tertiary care hospital in Karimnagar, India. Int J Basic Clin Pharmacol. 2018;7(4):807-12.

Cite this article as: Kalyani SSA, Srihitha $\mathrm{P}$, Sharma KA, Dharanija P, Bheemreddy SK. A prospective observational study on incidence of adverse drug reactions in a tertiary care teaching hospital: a pharmacovigilance study. Int J Basic Clin Pharmacol 2019;8:2423-33. 